\title{
A Proposed Algorithm for Generating the Reed-Solomon Encoding Polynomial Coefficients over GF(256) for $\mathrm{RS}[255,223] 8,32$
}

\author{
Frimpong Twum \\ Department Of Computer \\ Science Kwame Nkrumah \\ University of Science and Technology, \\ Kumasi, Ghana \\ W. W. Oblitey \\ Department Of \\ ComputeScience Indiana \\ University of Pennsylvania,USA.
}

\author{
J. B. Hayfron-Acquah \\ Department Of \\ Computer Science \\ Kwame Nkrumah University of \\ Science and Technology, \\ Kumasi, Ghana \\ R. K. Boadi \\ Department Of Mathematics \\ Kwame Nkrumah \\ University of \\ Science and Technology, \\ Kumasi, Ghana.
}

\begin{abstract}
The ability to detect and correct data loss is of crucial importance to securing and recovering data stored on any storage facility (most importantly, the cloud). Reed-Solomon (RS) codeword is the most used for achieving this purpose. RS codeword is widely used for detecting and recovering data transmission errors as well as data loss in storage. This paper illustrates how the coefficients of the encoding polynomial needed for the generation of the RS codeword are generated. An efficient algorithm for generating the encoding polynomial coefficient is proposed. The algorithm is implemented in JAVA for Galois Field [GF(256)] with 32 parity shards $\operatorname{RS}[255,223] 8,32$ to obtain an array of 32 coefficients as follows: $\quad\{232,29,189,50,142,246,232,15,43,82,164$, $238,1,158,13,119,158,224,134,227,210,163,50,107$, $40,27,104,253,24,239,216,45\}$
\end{abstract}

\section{Keywords}

Reed Solomon Codes, Galois Field, Encoding Polynomial, Error detection and Correction

\section{INTRODUCTION}

The application of finite field (Galois Field) over the last few decades has been enormous especially in the areas of data communication and storage [1], [2]. Other usages have been as follows: encryption, and data compression. Reed Solomon (RS) codes which operates over Galois Fields has been used extensively for the detection and correction of errors that occurs during data transmission and data storage [3]. A study by Ref. [4] outline other application areas for RS codes as Voyager spacecraft, detecting and correcting data losses in wireless transmission, dealing with scratches on CD's, correcting scanning errors in QR codes among others. This paper presents a concise approach for generating the encoding polynomial used for the generation of the RS codeword that is used for the detection and correction of data transmission errors and data storage losses. The study also proposes an efficient algorithm for generating the encoding polynomial for the generation of the RS codeword based on $\mathrm{GF}(256)$ for RS[255,223]8,32.

\section{RELATED WORKS}

\section{WHAT IS A FIELD?}

A set of numbers is a field if it satisfies the following properties.

\section{Field Properties}

The real number system is primarily a set, for e.g. $\{a, b, c$, $\ldots\}$, on which the operations of addition and multiplication are defined in such a way that for all pair of real numbers there exist a unique sum and product that are also real numbers and thus exhibit properties as follows [5], [6].

1. Cummulative Laws

$$
a+b=b+a \text {-------------- addition. }
$$

E.g. $1+2=2+1$.

$$
\mathrm{ab}=\mathrm{ba}
$$
multiplication.

E.g. $1 * 2=2 * 1$.

2. Associative Laws

$(\mathrm{a}+\mathrm{b})+\mathrm{c}=\mathrm{a}+(\mathrm{b}+\mathrm{c})$ addition

E.g. $(1+2)+3=1+(2+3)$.

(ab) $\mathrm{c}=\mathrm{a}(\mathrm{bc})$ $(1 * 2) * 3=1 *(2 * 3)$.

3. Distributive Laws $a(b+c)=a b+a c$

E.g. $1(2+3)=(1 * 2)+(1 * 3)$

There are distinct real numbers 0 and 1 such that

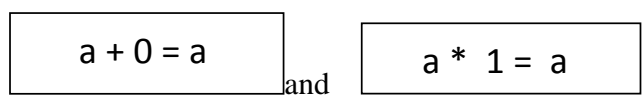

NB: For addition, the identity is ' 0 '. Whereas multiplication identity is 1 .

1. For each ' $a$ ' there is a real number ' $-\mathrm{a}$ ' such that $a+(-a)=0$ and if $a \neq 0$ there is a real number 
$\frac{1}{a}\left(\right.$ or $\left.a^{-1}\right)$ such that

$\mathrm{a}\left(\frac{1}{a}\right)=1$

2. $a+b \in R$, and $a * b \in R$ (closure laws), Example

For a given set to be a field it should satisfy all the field properties.

An a example of a field is the set of rational numbers.

i.e.

$$
\begin{aligned}
& a+b \in Q \quad \text { and } \quad a^{*} b \in Q . \\
& \text { e.g. if } a=2 \\
& a+(-a)=0 \quad \text { and } \\
& a\left(\frac{1}{a}\right)=1
\end{aligned}
$$

\section{What is A Finite Field?}

A finite field (aka. Galois Field - GF) is a field with finitely defined elements where upon performing the arithmetic operations of addition, subtraction, division, or multiplication of $f(p) \bmod p$ on any two of the field elements, the result is always an element in the set.

$\mathrm{F}_{\mathrm{p}}=\{0,1,2, \ldots, \mathrm{p}-1\}$

$\bullet,+$ : integer addition and multiplication in modulo $\mathrm{p}$

This property of a finite field enables its usage for error detection and data recovery in data communication and data storage [5], [6].

A finite field is constructed using a prime number base or powers of a prime number. This is to ensure a unique value is obtained when addition and multiplication operations are performed on any two of the field elements. For example, finite field elements for $\mathrm{GF}(2)$ is constructed as $\{0,1\}, \mathrm{GF}(3)$ as $\{0,1,2\}$, and $\mathrm{GF}(7)$ as $\{0,1,2,3,4,5,6\}[7]$.

In the case of the powers of prime, a finite field for $\operatorname{GF}\left(2^{\mathrm{n}}\right)$, where 2 is the prime base and ' $n$ ' is the exponent determines the number of elements in the field.

As an example $\operatorname{GF}\left(2^{3}\right)$ which is the same as $\operatorname{GF}(8)$ has field elements as $\{0,1,2,3,4,5,6,7\}$, and $\mathrm{GF}(16)$ represented in prime powers of 2 as $\operatorname{GF}\left(2^{4}\right)$ has elements as $\{0,1,2,3,4,5$, $6,7,8,9,0,11,12,13,14,15\}$. It follows therefore that $\mathrm{GF}(\mathrm{N})=\mathrm{GF}\left(2^{\mathrm{n}}\right)$ has field elements as $\{0,1,2, \ldots, \mathrm{n}-1)$. Hence $\mathrm{GF}\left(2^{8}\right)=\mathrm{GF}(256)$ has 256 field elements as $\{0,1,2,3, \ldots$, $255\}$. This is an example of a modulus 256 field and hence 255 is the maximum value [2], [7], [8].

For computer computational operations, a base 2 prime base is used for representing the field elements as prime powers [4]

The elements of a finite field are usually represented as polynomials that take their coefficients from a particular field $F_{p}$. For example, for a polynomial, $F_{p}(x)=a_{0}+a_{1} x+a_{2} x^{2}+\ldots$ $+a_{n} x^{n}$ where $a_{i} \in F$. A deg. 1 polynomial of $F_{2}(x)=a_{0}+a_{1} x$, has field elements represented using alpha powers as $\{\alpha, 1+$ $\alpha\}$ which are already in their irreducible form. Elements of deg. 2 polynomials in $\mathrm{Fp}(\mathrm{x})=\mathrm{a}_{0}+\mathrm{a}_{1} \mathrm{x}+\mathrm{a}_{2} \mathrm{x}^{2}$ are obtained as $\left\{\alpha^{2}, \alpha^{2}+\alpha, \alpha^{2}+1, \alpha^{2}+\alpha+1\right\}$ [2], [9].

Similarly for deg. 3 polynomials in $\mathrm{Fp}(\mathrm{x})=\mathrm{a}_{0}+\mathrm{a}_{1} \mathrm{x}+\mathrm{a}_{2} \mathrm{x}^{2}+$ $\mathrm{a}_{3} \mathrm{x}^{3}$ the elements are as follows $\left\{\alpha^{3}, \alpha^{3}+\alpha^{2}, \alpha^{3}+\alpha^{2}+\alpha, \alpha^{3}+\right.$ $\left.1, \alpha^{3}+\alpha^{2}+1, \alpha^{3}+\alpha+1, \alpha^{3}+\alpha, \alpha^{3}+\alpha^{2}+\alpha+1\right\}$.

\section{Galois Field Elements Construction}

Galois field represented as binary form is very convenient for detecting and correcting errors (in transmission or storage) and as well as for ciphering computer data. This is because it is a finite field and adheres to properties of a field. The elements of Galois field, $\mathrm{GF}\left(\mathrm{P}^{\mathrm{m}}\right)$ is defined as

$$
\begin{aligned}
& \mathrm{F}_{\mathrm{p}}{ }^{\mathrm{m}}=\left\{\mathrm{a}_{0}+\mathrm{a}_{1} \mathrm{x}+\mathrm{a}_{2} \mathrm{x}^{2}+\ldots+\mathrm{a}_{\mathrm{m}-1} \mathrm{x}^{\mathrm{m}-1}\right\} \text { where } \mathrm{a}_{\mathrm{i}} \in \mathrm{F}_{\mathrm{p}} . \\
& +: \text { addition in } \mathrm{F}_{\mathrm{p}}(\mathrm{x}) \bmod \mathrm{p} \\
& \bullet: \text { multiplication in } \mathrm{F}_{\mathrm{p}}(\mathrm{x}) \bmod \pi(\mathrm{x})
\end{aligned}
$$

Where $\pi(\mathrm{x})$ := deg.m irreducible polynomial in $\mathrm{F}_{\mathrm{p}}(\mathrm{x})$.

Irreducible polynomials (polynomial that cannot be factord)for example $x^{2}+1$ has no roots and are used to construct the elements of $\mathrm{GF}\left(2^{\mathrm{n}}\right)$. Reducible polynomials for example, $x^{2}-1$, has roots as -1 and +1 and hence are not used when generating the elements of $\mathrm{GF}\left(2^{\mathrm{n}}\right)$. As an example, given $F_{p}=F_{4}=F_{2}^{2}$, the elements of polynomials of deg $\leq \mathrm{m}-1$ with coefficients from $\mathrm{F}_{\mathrm{p}}$ are given as $\mathrm{F}_{\mathrm{p}}{ }^{\mathrm{m}}=\mathrm{F}_{2}{ }^{2}=\{0,1, \alpha, 1+$ $\alpha\}, p=2$, and $\mathrm{m}=2$ [8], [9]. For $\mathrm{F}_{2}{ }^{3}$ the field elements in powers of alpha are obtained as follows:

$$
\begin{array}{lcc}
\text { deg. } 0 & \text { deg.1 } & \text { deg. } 2 \\
\mathrm{a}_{0} & \mathrm{a}_{0}+\mathrm{a}_{1} \alpha & \mathrm{a}_{0}+\mathrm{a}_{1} \alpha+\mathrm{a}_{2} \alpha^{2} \\
\mathrm{~F}_{\mathrm{p}}{ }^{\mathrm{m}}=\mathrm{F}_{2}{ }^{3}=\left\{0,1, \alpha, \alpha+1, \alpha^{2}, \alpha^{2}+\alpha, \alpha^{2}+1, \alpha^{2}+\alpha+1\right\}, \text { where } \\
\mathrm{p}=2, \text { and } \mathrm{m}=3 .
\end{array}
$$

\section{Galois Field (Gf) Arithmetics}

Arithmetic in GF or finite field is different from standard arithmetic. Unlike standard arithmetic, which has an infinite number of elements, there is limited number of elements in a finite field.

Thus, arithmetic in finite field is basically carried out on a set of elements in which when the arithmetic operations of addition multiplication subtraction, or division is performed on the set the results is always found in the same set [9], [10].

Recall, a finite field of elements $\mathrm{P}^{\mathrm{n}}$ is basically represented in Galois field as $\operatorname{GF}\left(\mathrm{P}^{\mathrm{n}}\right)$, where $\mathrm{P}$ is a prime base and $\mathrm{n}$ is the exponent of $\mathrm{P}$, modulus $\mathrm{P}$. For example, $\mathrm{GF}(8)=\mathrm{GF}\left(2^{3}\right)$ modulus 8 and the elements in the field are $\{0,1,2,3,4,5,6$, $7\}$.

\section{Addition and Subtraction In Gf(8)}

The steps to performing addition and subtraction in $\mathrm{GF}\left(2^{3}\right)$ are as follows:

- the polynomials of $\mathrm{deg} \leq \mathrm{m}-1$ with coefficients from $\mathrm{F}_{2}{ }^{3}$, where $\mathrm{m}=3$ and $\mathrm{p}=2$ is defined to obtain the field elements for $\mathrm{F}_{2}{ }^{3}$ as in section 2.3 above as: Elements of $F_{2}^{3}=\left\{0,1, \alpha, \alpha+1, \alpha^{2}, \alpha^{2}+\alpha, \alpha^{2}+1, \alpha^{2}+\right.$ $\alpha+1\}$

- the addition table is constructed using the resulting elements of $\mathrm{F}_{2}{ }^{3}$ in modulus 2 as follows:

The GF addition table of Table 1 also has the entries for the GF subtraction operation as subtraction is performed as addition in computer systems using the additive inverse of the subtrahend. An element's additive inverse is the element that results with zero when added to the minuend. Rule: $a+(-a)=$ 0

\section{Multiplication \& Division In Gf(8)}

- The multiplication table for $G F(8) \bmod 2$ is constructed using the elements of $\mathrm{F}_{2}{ }^{3}$ and a deg.3 irreducible primitive polynomial in $\mathrm{F}_{2}{ }^{3}$ obtained as 
$\alpha^{3}+\alpha+1$ or $\alpha^{3}+\alpha^{2}+1$. This resulted with the field elements for $\mathrm{F}_{2}{ }^{3}$ as shown in powers of alpha and is used for the construction of the multiplication table, Table 2 .

$\mathrm{F}_{2}^{3}=\left\{0,1, \alpha, \alpha^{2}, \alpha^{3}, \alpha^{4}, \alpha^{5}, \alpha^{6}\right\}, \bmod 2$

Using the irreducible polynomial $\alpha^{3}+\alpha+1 \alpha^{3}=\alpha+1 \alpha^{4}=\alpha\left(\alpha^{3}\right)$ $=\alpha(\alpha+1)=\alpha^{2}+\alpha$

$\alpha^{5}=\alpha\left(\alpha^{4}\right)=\alpha\left(\alpha^{2}+\alpha\right)=\alpha^{3}+\alpha^{2}$, Now Substituting for $\alpha^{3}=\alpha+1$ gives

$\alpha^{5}=\alpha^{2}+\alpha+1$

$\alpha^{6}=\alpha\left(\alpha^{5}\right)=\alpha\left(\alpha^{2}+\alpha+1\right)=\alpha^{3}+\alpha^{2}+\alpha$, Now Substituting for $\alpha^{3}$ gives $\left(\alpha+1+\alpha^{2}+\alpha\right) \bmod 2 \quad \therefore \alpha^{6}=\alpha^{2}+1$

$\alpha^{7}=\alpha\left(\alpha^{6}\right)=\alpha\left(\alpha^{2}+1\right)=\alpha^{3}+\alpha$ Substituting for $\alpha^{3}$ gives $(\alpha$ $+1+\alpha) \bmod 2=1$

$\alpha^{8}=\alpha\left(\alpha^{7}\right)=\alpha(1)=\alpha, \quad \alpha^{9}=\alpha^{2}, \quad \alpha^{10}=\alpha^{3}, \alpha^{11}=\alpha^{4}, \quad \alpha^{12}=$ $\alpha^{5}, \quad \alpha^{13}=\alpha^{6}$

As can be seen, the element values for alpha repeats from $\alpha^{7}$ indicating $\mathrm{GF}(8)$ is a field.

Table 2 also present entry values for division in $\mathrm{GF}(8)$ which is performed using multiplicative inverse of the elements in the set. Rule: $\mathbf{a}^{*}\left(\mathbf{a}^{-1}\right)=\mathbf{1}$

As an example dividing $\alpha^{5}$ by $\alpha^{3}$ or (7/3) imply multiplying $\alpha^{5}$ by the multiplication inverse of $\alpha^{3}$ as follows: From Table 2, the multiplication inverse of $\alpha^{3}$ is the corresponding element in the matrix that when multiplied by $\alpha^{3}$ gives 1 as the result (i.e. Rule: $\left.\mathbf{a} *\left(\mathbf{a}^{-1}\right)=\mathbf{1}\right)$.

Hence the inverse of $\alpha^{3}$ is $\alpha^{4}=6$. Therefore $\alpha^{5} / \alpha^{3}$ is obtained $\left(\alpha^{5 *} \alpha^{4}=\alpha^{2}\right)$. Thus: $7 / 3$ implies $7 * 6=4$ [where 6 is the inverse of 3].

It can be seen from the Table 1 and Table 2 there are no identical entry in any of the rows or columns and there are also no repeating or negative entries in any row or column. These characteristics of the field element set makes the use of Galois field ideal for data recovery and/or error detection in data communication and/or data storage. Any of the elements in the set can be regenerated from the rest of the elements in the event of loss or damage. This is useful particularly in distributed data storage as cloud computing as in the event of a system breakdown or disk drives failures, the system can recover missing data and prevent any data loss. This system of data recovery is much efficient and cost effective than those of RAID technology [10].

\section{The RS Codeword}

The ability to detect and correct data loss is of crucial importance to security and recovering data stored on any storage facility (most importantly, the cloud). Reed-Solomon (RS) codeword is the most used for achieving this purpose. The following section illustrates how the RS codewords are generated and used for the detection and correction of errors in data transmission and storage.

According to Ref. [3], [9] the RS codeword is generated using three (3) polynomials namely:

The "Irreducible Polynomial" (i.e. the polynomial equivalent of a prime number) is used as the generating polynomial for the Galois field elements generation. For the GF (8) elements generation, the irreducible polynomial $\left(\boldsymbol{\alpha}^{3}+\boldsymbol{\alpha}+\mathbf{1}\right)$ is used.
The "Generator Polynomial" - This polynomial is required for generating the encoding polynomial (which is the $3^{\text {rd }}$ polynomial needed for the generation of the RS codeword). The generator polynomial is a generic polynomial of the form; $\mathbf{G}(\mathbf{x})=\left(\mathbf{x}-\boldsymbol{\alpha}^{1}\right)\left(\mathbf{x}-\boldsymbol{\alpha}^{2}\right)\left(\mathbf{x}-\boldsymbol{\alpha}^{3}\right) \ldots\left(\mathbf{x}-\boldsymbol{\alpha}^{2 \mathbf{t}}\right)$, where $\alpha^{1}, \alpha^{2}, \alpha^{3}$, etc. are the field elements and the value $2 t$ determines the number of the Forward Error Correction (FEC) require.

For example, assuming an RS codeword of $\mathbf{R S}[\mathbf{7 , 5}] \mathbf{S = 3}, \mathbf{t}=\mathbf{2}$ where ' $\mathrm{S}$ ' is the number of bits making a symbol size (in this case 3-Bit symbols), ' $t$ ' is the number of the 3-Bits symbols used for error correction (in this case 2 (3-Bits) symbols), and 5 is the number of 3-Bits symbols used for representing the actual data chunks, whiles 7 is the total number of RS codewords for a GF (8). The generic expression (a.k.a. Maximum Distance Separable-MDS) for RS codeword is given as $\mathbf{R S}[\mathbf{n}, \mathbf{k}] \mathbf{s}, \mathbf{t}$, where $\mathrm{n}$ is the number of codewords given as $2^{\mathrm{s}}-1$ and $\mathrm{k}$ is the number of data chunks.

Encoding Polynomials - for the RS[7,5]3,2 specification codeword example, 2 symbols are needed for FEC and hence only 2 of the Generator Polynomials are required as follows: $G(x)=\left(x-\alpha^{1}\right)\left(x-\alpha^{2}\right)$.

Since addition and subtraction operations give the same result in GF, $\mathbf{G}(\mathbf{x})=\left(\mathbf{x}+\boldsymbol{\alpha}^{1}\right)\left(\mathbf{x}+\boldsymbol{\alpha}^{2}\right)$. From the Table 1, $\boldsymbol{\alpha}^{1}=\mathbf{2}$, and $\alpha^{2}=4$ therefore $G(x)=(x+2)(x+4)$. Hence, $G(x)=x^{2}+4 x+$ $2 x+8=x^{2}+6 x+8 \quad 8$ in binary is 1000 which is bigger than the largest field elements of 7 therefore the Generating polynomial of 1011 is XOR.

1000

$\underline{\mathrm{XOR} 1011}$

$0011=3$.

Therefore, $\mathbf{G}(\mathbf{x})=\mathbf{x}^{\mathbf{2}}+\mathbf{6} \mathbf{x}+\mathbf{3}$ is the encoding polynomial which is expressed also as 163 and is used for the RS codeword generation.

\section{METHODOLOGY GENERATION OF COEFFICIENT VALUES OF THE ENCODING POLYNOMIAL}

This study is aimed at proposing an efficient algorithm for the generation of the encoding polynomial coefficients for $\mathrm{GF}(256)$ that are needed for the generation of the RS codeword of RS[255,223]8,32. To achieve this aim require 3 polynomials as follows:

- The irreducible polynomial (also referred to as the generating polynomial)

- The generator polynomial

- The encoding polynomial

As noted in the case of $\mathrm{GF}(8)$ earlier in the literature review, for $\mathrm{GF}(256)$ or $\mathrm{GF}\left(2^{8}\right)$ (the focus of this study), the number of RS codewords generated is obtained as $n=2^{8}-1=255$. Hence for the 32 (8-bits Symbols) parity shards or (32 Forward Error Correction (FEC) codes) require by this study imply splitting files into 223 (8-bits symbols) data shards. To achieve this, the following are undertaken.

Step-1: A degree 8 irreducible polynomial (a polynomial equivalent of a prime number) in $\mathrm{F}_{2}{ }^{8}$ obtained as $\mathrm{P}(\mathrm{x})=\alpha^{8}+$ $\alpha^{4}+\alpha^{3}+\alpha^{2}+1=285$ [2], [11], is used to generate the $\operatorname{GF}(256)$ field elements of $(0-255)$.

Step-2: The generator polynomial which is needed for the generation of the encoding polynomial is defined for the 
creation of 32 (8-bits Symbols) parity shards or (32 Forward Error Correction (FEC) codes) as follows: $\mathbf{G}(\mathbf{x})=\left(\mathbf{x}-\boldsymbol{\alpha}^{\mathbf{1}}\right)(\mathbf{x}-$ $\left.\alpha^{2}\right)\left(x-\alpha^{3}\right)\left(x-\alpha^{4}\right)\left(x-\alpha^{5}\right)\left(x-\alpha^{6}\right)\left(x-\alpha^{7}\right)\left(x-\alpha^{8}\right)\left(x-\alpha^{9}\right)\left(x-\alpha^{10}\right)(x-$ $\left.\alpha^{11}\right)\left(x-\alpha^{12}\right)\left(x-\alpha^{13}\right)\left(x-\alpha^{14}\right)\left(x-\alpha^{15}\right)\left(x-\alpha^{16}\right)\left(x-\alpha^{17}\right)\left(x-\alpha^{18}\right)\left(x-\alpha^{19}\right)$ $\left(\mathrm{x}-\alpha^{20}\right)\left(\mathrm{x}-\alpha^{21}\right)\left(\mathrm{x}-\alpha^{22}\right)\left(\mathrm{x}-\alpha^{23}\right)\left(\mathrm{x}-\alpha^{24}\right)\left(\mathrm{x}-\alpha^{25}\right)\left(\mathrm{x}-\alpha^{26}\right) \quad(\mathrm{x}-$ $\left.\alpha^{27}\right)\left(\mathrm{x}-\alpha^{28}\right)\left(\mathrm{x}-\alpha^{29}\right)\left(\mathrm{x}-\alpha^{30}\right)\left(\mathrm{x}-\alpha^{31}\right) \quad\left(\mathrm{x}-\alpha^{32}\right)$ ----[expression 1]

Now substituting values of ' $\boldsymbol{\alpha}$ ' in Step-1 with their decimal equivalent from the $\mathrm{GF}(256)$ elements

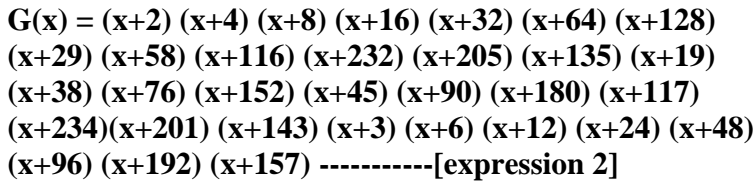

Since the addition and subtraction arithmetic operation in GF gives the same results, the subtraction operator in [expression $1]$ is replaced with addition in [expression 2].

Step-3: The generator polynomial of [expression 2] is then expressed in the form

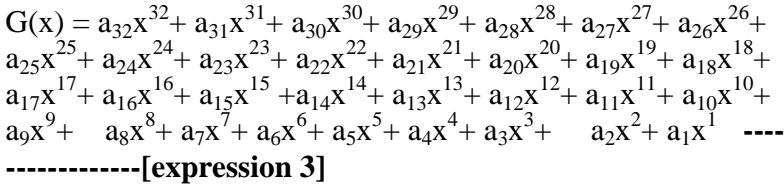

Where the coefficient values $\left(\mathrm{a}_{32}, \mathrm{a}_{31}, \mathrm{a}_{30}, \mathrm{a}_{29}, \mathrm{a}_{28}, \mathrm{a}_{27}, \mathrm{a}_{26}, \mathrm{a}_{25}, \mathrm{a}_{24}, \mathrm{a}_{23}, \mathrm{a}_{22}, \mathrm{a}_{21}, \mathrm{a}_{20}, \mathrm{a}_{19}, \mathrm{a}_{18}, \mathrm{a}_{17}, \mathrm{a}_{16}, \mathrm{a}_{15}\right.$, $\left.\mathrm{a}_{14}, \mathrm{a}_{13}, \mathrm{a}_{12}, \mathrm{a}_{11}, \mathrm{a}_{10}, \mathrm{a}_{9}, \mathrm{a}_{8}, \mathrm{a}_{7}, \mathrm{a}_{6}, \mathrm{a}_{5}, \mathrm{a}_{4}, \mathrm{a}_{3}, \mathrm{a}_{2}, \mathrm{a}_{1} \mathrm{x}^{1}\right)$ are used for the generation of the encoding polynomial which is used for the generation of the RS codeword for error detection and recovery in the vent of data loss, damage, or alteration in transmission or in storage. The algorithm proposed by this study for the generation of the coefficient values is as explained below.

\section{ALGORITHM FOR THE GENERATION OF THE COEFFICIENTS OF THE ENCODING POLYNOMIAL}

The Generator polynomial is of the form

$$
g(x)=\left(x+\alpha^{1}\right)\left(x+\alpha^{2}\right)\left(x+\alpha^{3}\right) \ldots\left(x+\alpha^{n}\right)
$$

where $n$ is the number of parity shards

The coefficients of $x$ in the expansion of $g(x)$ is found using the algorithm below

$$
\begin{aligned}
& \text { for pow }=0 \text { to } n \\
& \rightarrow \text { sum all the combinations of the set }\left\{\alpha^{1}, \alpha^{2}, \alpha^{3} \ldots \alpha^{n}\right\} \\
& \text { that have pow elements }
\end{aligned}
$$

For example, to find the coefficients for $n=4$, assuming the Galois Field elements are

$$
\begin{array}{ll}
\text { - } & \alpha^{1}=a \\
\text { - } & \alpha^{2}=b \\
\text { - } & \alpha^{3}=c \\
\text { - } & \alpha^{4}=d
\end{array}
$$

Then the looping process perform the following action

- $\quad p o w=0:\{\}$

- $\quad$ pow $=1: a+b+c+d$

- $\quad p o w=2: a b+a c+a d+b c+b d+c d$
- $\quad p o w=3: a b c+a b d+a c d+b c d$

Thus

1. Loop $n+1$ times $(i \rightarrow 0$ to $n)$

a. Generate all subsets of the alpha exponents set, of size $(n-i)$

b. Multiply the components of each of the subsets

c. Add the products of the subset components

d. Save the sum of the subsets in a coefficients array

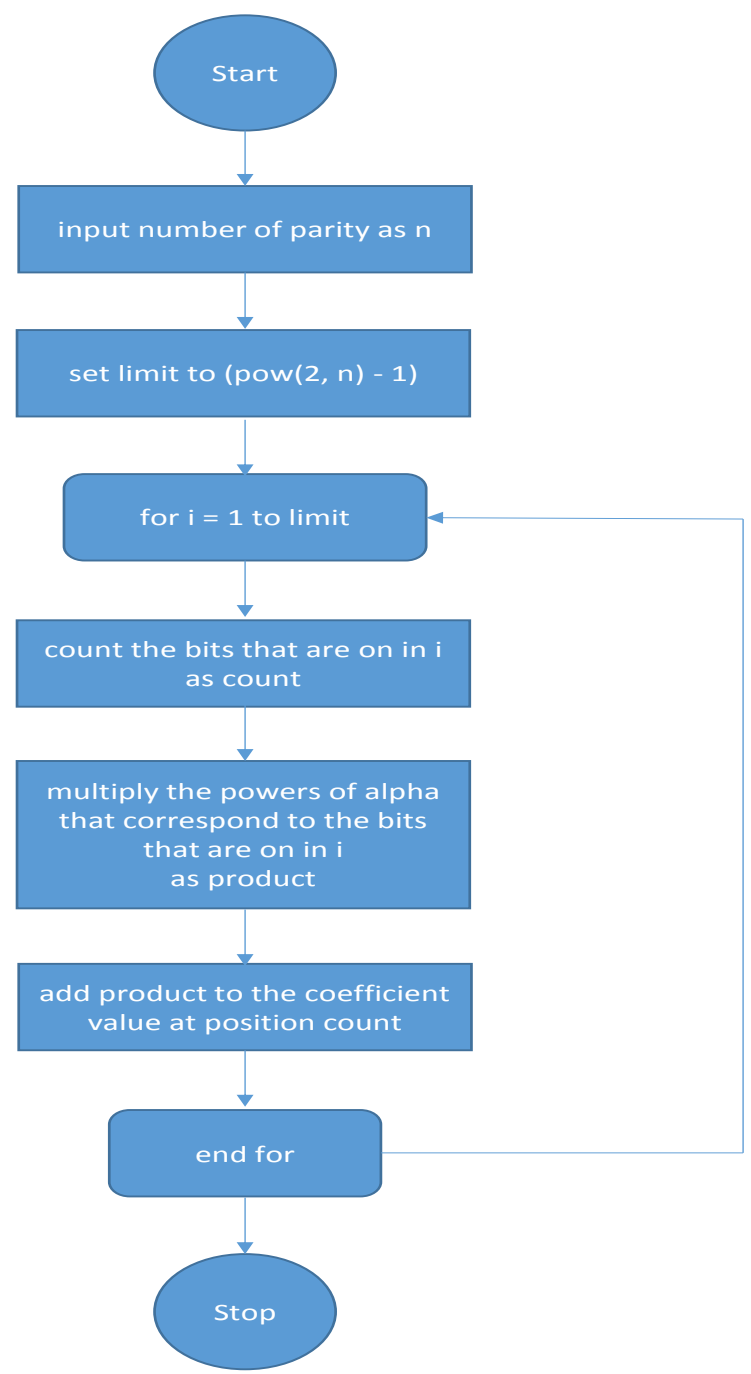

Figure 1: Flow diagram for the algorithm

\section{IMPLEMENTATION}

\section{GENERATING THE GF(256) FIELD ELEMENTS}

The process of representing a finite field in a computer, especially for arithmetic purposes has been refined. Representing the elements of $\mathrm{GF}(8)$ like this $\{0,1,2,3,4,5$, $6,7\}$ for example is more difficult to implement than like this $\{0,1,2,4,3,6,7,5\}$. Doing arithmetic in GF by hand is not much of a problem. However by using a computer the elements of the field are best represented as exponents of 2 . An irreducible polynomial is used as a modulus to ensure the exponents of 2 do not repeat. 
This example demonstrates the use of the irreducible polynomial 29(i.e. 285-256) to keep the powers of 2 within the range $0-255$

$$
\begin{aligned}
& * * \mathrm{a}^{\wedge} 0=2^{\wedge} 0=1 \\
& * * \mathrm{a}^{\wedge} 1=1 * 2=2 \\
& * * \mathrm{a}^{\wedge} 2=2 * 2=4 \\
& * * \mathrm{a}^{\wedge} 3=4 * 2=8 \\
& * * \mathrm{a}^{\wedge} 4=8 * 2=16 \\
& * * \mathrm{a}^{\wedge} 5=16 * 2=32 \\
& * * \mathrm{a}^{\wedge} 6=32 * 2=64 \\
& * * \mathrm{a}^{\wedge} 7=64 * 2=128 \\
& * * \mathrm{a}^{\wedge} 8=128 * 2=256
\end{aligned}
$$

Since 256 is outside the range $0-255$, subtract 256 from it to put it back within range, then XOR with the irreducible polynomial (29) to get a unique start value.

Hence $\mathrm{a}^{\wedge} 8=(256-256) \oplus 29=29$

$$
\begin{aligned}
& * * \quad a^{\wedge} 9=29 * 2=58 \\
& * * \quad a^{\wedge} 10=58 * 2=116 \\
& * * a \wedge 11=116 * 2=232 \\
& * * \\
& a^{\wedge} 12=232 * 2=464
\end{aligned}
$$

Once again, 464 is outside the range so subtract 256 to put it back within range, then add 29 to get a unique number. Hence the calculation continues as

$$
\therefore \mathrm{a}^{\wedge} 12=(464-256) \oplus 29=205
$$

\begin{tabular}{cc}
464 & \\
-256 & \\
\hline $208=$ & 11010000 \\
$\oplus 29=$ & 11101 \\
\hline & $11001101=205$
\end{tabular}

$$
\begin{aligned}
& * * \quad a^{\wedge} 13=205 * 2=410===(410-256) \oplus 29=135 \\
& * * a^{\wedge} 14=135 * 2=270===(270-256) \oplus 29=19 \\
& * * a^{\wedge} 15=19 * 2=38 \\
& * * a \wedge 16=38 * 2=76
\end{aligned}
$$

As can be seen, by repeatedly subtracting 256 from any product value that falls outside of the range of 0-255 and XOR with the irreducible polynomial of 29 the process keeps generating unique numbers within the $0-255$ range resulting with the GF(256) table (See Table 3).

\section{IMPLEMENTING ARITHMETIC OPERATION IN GF ADDITION AND SUBTRACTION IN GF}

Additions and subtractions in a Galois Field both come down to the bitwise XOR operation because in $\mathrm{GF}(2)$

$$
\begin{array}{llll}
\text { a. } 1+1=0 & --> & 0-1=1 \\
\text { b. } 1+0=1 & --> & 1-1=0 & \text { AND } 1-0=1 \\
\text { c. } 0+0=0 & --> & 0-0=0
\end{array}
$$

Per the definition of the XOR operation, the result is 0 when the operands are alike and 1 when the operands are different. That definition is satisfied by both addition and subtraction in $\mathrm{GF}(2)$.
Thus the implementation of the addition and subtraction functions in Java code is simply to XOR the arguments.

\section{MULTIPLICATION AND DIVISION IN GF}

Multiplication contains an element of addition in it (in fact, multiplication is simply repeated addition) but since addition is implemented as an XOR operation in $\mathrm{GF}(2)$, repeated addition will always result in an answer of 0 .

An alternative is to use this addition feature of logarithms

$$
\mathrm{A} * \mathrm{~B}=\log \text {-inverse }(\log (\mathrm{A})+\log (\mathrm{B}))
$$

Division in the Galois Field is also implemented in Java using logarithms

$$
\mathrm{A} / \mathrm{B}=\log \text {-inverse }(\log (\mathrm{A})-\log (\mathrm{B})) ;
$$

\section{LOGARITHMS and EXPONENTS}

The precondition for using the addition and subtraction features of logarithms in the multiplication and division in Galois Fields is that the log values for all the elements of the field must first be known. Fortunately the method used earlier to generate the elements of the Galois Field uses exponents of 2.

Thus the log of any element in the field is the exponent of 2 (or exponent $\boldsymbol{\alpha}$ as in Table 1) indexed to the position corresponding to its generated exponent value. For example, $2^{\wedge} 3$ generated 8 , so $\log (8)=3$, and $2^{\wedge} 7$ generated 128 , and hence $\log (128)=7$, in $\operatorname{GF}(256)$.

\section{IMPLEMENTING THE ALGORITHM FOR THE GENERATION OF COEFFICIENTS OF THE ENCODING POLYNOMIAL GENERATION ALGORITHM}

Both the encoding and decoding processes of the ReedSolomon algorithm rely on a generator polynomial of the form

$$
g(x)=\prod_{i=1}^{n}\left(x-\alpha^{i}\right)
$$

where $n$ is the number of parity shards.

It should be noted that with Galois Field arithmetic, addition and subtraction both result in the same XOR operation. As such, the algorithmic representation of the above formula used the Galois Addition method.

It was determined that the coefficients resulting from the expansion of the encoding polynomial formula

$$
g(x)=\sum_{i=0}^{n} c_{i} x^{n-i}
$$

followed this pattern

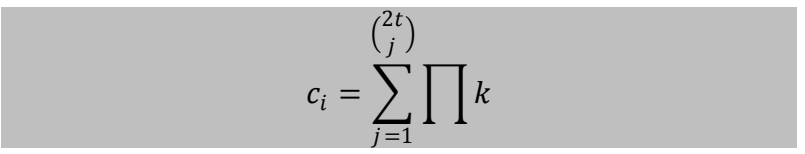

where $k$ is a member of one subset of the Galois Field's elements that has $i$ members

The algorithm focused on determining the coefficients only. This involved finding all the possible combinations of the first 2t Galois Field Elements and performing the appropriate multiplication and addition operations on them. The process used is described in greater detail below: 
1. Obtain the first $2 t$ values in the Galois field and save them in an array

2. Determine the highest integer (upper limit) that has $2 t$ bits using the formula below

$$
\text { limit }=2^{2 t}-1
$$

3. Create $2 t$ masks for determining which bits are on in any given integer that is within the range $[0$, limit $]$.The masks are simply integer values whose bit representations have only one bit on and all other bits off. In other words, the masks are the powers of 2 from $2^{0}$ to $2^{2 t}$. When a bitwise $A N D$ operation is performed with a number and any of the masks, a result of 0 means that the particular bit which the mask has on, is off in the number. However, a result greater than 0 indicates that that particular bit is on. Using this approach, it is possible both to determine which particular bits are on in the number and also count them.

4. Create $2 t$ accumulators, initialized to 0 . These accumulators will hold the values of the coefficients when the program runs to a completion.

5. The combinations of the first $2 t$ Galois Field elements are generated as integer values from 1 to limit. Thus, a loop is used which runs from 1 to limit. For each integer in the range

a. The masks are applied to the integer to determine which bits are on.

b. The Galois Field elements whose positions corresponds to the bits that are on in the integer, are multiplied to get the

\section{RESULTS}

This algorithm was applied to $G F(256,285)$ with $n=32$.

The resulting values of coefficient[] obtained from a Java code implementation are as follows: product of all the elements in that particular combination.

c. Concurrently, the number of bits that are on are counted. The product from multiplying the bits that are on, is added to the accumulator at the position of the count. This ensures that all the products derived from multiplying combinations of a particular length are accumulated in a single accumulator.

\section{PSEUDOCODE FOR THE COEFFICIENT GENERATION ALGORITHM}

1. $\quad$ Iimit $=2^{n}-1$

2. for $i=1$ to limit

a. $\quad$ product $=1$
b. $\quad$ for $j=1$ to $n$

$$
\begin{gathered}
\text { i. } \quad x=i \text { XOR mask }[j] \\
\text { ii. } \quad \text { if } x>1 \\
\text { 1. } \quad \text { product }= \\
\text { product } * \text { exp }[\text { count }] \\
\text { 2. count }+1 \\
\text { coefficient }[\text { count }]= \\
\text { coefficient }[\text { count }]+\text { product }
\end{gathered}
$$

c. coefficient $[$ count $]=$

- The array mask[] contains the powers of two from 1 to $2^{n}$

- The array exp野 contains the Galois field elements

- The array coefficient[] holds the values of the coefficients at the end of the program execution

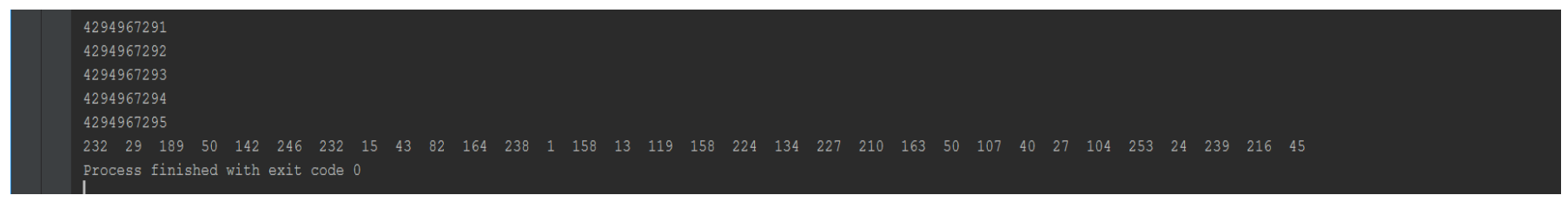

\section{CONCLUSION}

This research provides overview of the Galois field theory and Reed Solomon Coding. It proposes and implements an algorithm for the generation of the encoding polynomial coefficients that are used for the Reed Solomon codeword generation. The outcome of this study enables the generation of the Reed Solomon codeword that is used for detection and correction of data loss in transmission or storage.

\section{REFERENCES}

[1] Plank, J. S. (2013). Erasure Codes for Storage Systems. Available at:

https://www.usenix.org/system/files/login/articles/10_pla nk-online.pdf

[2] REDTITAN, (2011). Error detection and correction. Available at: http://www.pclviewer.com/rs2/galois.html

[3] cs.cmu.edu (1998). Reed-Solomon Codes. An introduction to Reed-Solomon codes: principles, architecture and implementation. Available at: https://www.cs.cmu.edu/ guyb/realworld/reedsolomon/r eed_solomon_codes.html

[4] Cox, R. (2012). Finite Field Arithmetic and ReedSolomon Coding. Available at: http://research.swtch.com/field

[5] Trench W. F., (2003). Introduction to Real Analysis. Library of Congress Cataloging-in-Publication Data. Available at: http://ramanujan.math.trinity.edu/wtrench/texts/TRENC H_REAL_ANALYSIS.PDF

[6] Wang, J. (2009). Computer Network Security Theory and Practice. Springer

[7] Benvenuto, C. J. (2012). Galois Field in Cryptography. Available at: https://www.math.washington.edu/ morrow/336_12/pap ers/juan.pdf 
[8] Lynson, B. Tutorial on Reed-Solomon Error Correction Coding. NASA Tech Brief MSC-21834. Available at: http://jeffareid.net/misc/msc-21834.pdf

[9] Hill, T. (2013). Reed Solomon Codes Explained. Available at: https://www.tonyhill.info/app/download/.../Reed+Solomon+Explained+ V1-0.pdf

[10] Plank, J. S. (1997). A Tutorial on Reed-Solomon Coding for Fault-Tolerance in RAID-like Systems. SoftwarePractice and Experience. Vol.27, No.9, 995-1012. Available at: http://cgi.di.uoa.gr/ ad/M155/Papers/RSTutorial.pdf

\section{APPENDIX}

Table 1: Addition in GF(8) $\bmod 2$

\begin{tabular}{|c|c|c|c|c|c|c|c|c|}
\hline+ & $\mathbf{0}$ & 1 & $\begin{array}{c}\boldsymbol{\alpha} \\
=2\end{array}$ & $\begin{array}{c}\alpha^{3} \\
\alpha+\mathbf{1}=\mathbf{3}\end{array}$ & $\begin{array}{l}\alpha^{2} \\
=4\end{array}$ & $\begin{array}{c}\alpha^{4} \\
\alpha^{2}+\alpha=6\end{array}$ & $\begin{array}{c}\alpha^{6} \\
\alpha^{2}+\mathbf{1}=\mathbf{5}\end{array}$ & $\begin{array}{c}\alpha^{5} \\
\alpha^{2}+\alpha+1=7\end{array}$ \\
\hline $\mathbf{0}$ & 0 & 1 & 2 & 3 & 4 & 6 & 5 & 7 \\
\hline 1 & 1 & 0 & 3 & 2 & 5 & 7 & 4 & 6 \\
\hline $\begin{array}{c}\alpha \\
=2\end{array}$ & 2 & 3 & 0 & 1 & 6 & 4 & 7 & 5 \\
\hline $\begin{array}{c}\alpha^{3} \\
\boldsymbol{\alpha}+\mathbf{1}=\mathbf{3}\end{array}$ & 3 & 2 & 1 & 0 & 7 & 5 & 6 & 4 \\
\hline $\begin{array}{l}\alpha^{2} \\
=4\end{array}$ & 4 & 3 & 6 & 7 & 0 & 2 & 1 & 3 \\
\hline $\begin{array}{c}\alpha^{4} \\
\alpha^{2}+\alpha=6\end{array}$ & 6 & 7 & 4 & 5 & 2 & 0 & 3 & 1 \\
\hline $\begin{array}{c}\alpha^{6} \\
\boldsymbol{\alpha}^{2}+\mathbf{1}=\mathbf{5}\end{array}$ & 5 & 4 & 7 & 6 & 1 & 3 & 0 & 2 \\
\hline $\begin{array}{c}\alpha^{5} \\
\alpha^{2}+\alpha+1=7\end{array}$ & 7 & 6 & 5 & 4 & 3 & 1 & 2 & 0 \\
\hline
\end{tabular}

Table 2: Multiplication in GF(8) $\bmod 2$

\begin{tabular}{|c|c|c|c|c|c|c|c|c|}
\hline$*$ & 0 & $\begin{aligned} & \alpha^{7} \\
= & 1\end{aligned}$ & $\begin{array}{c}\alpha \\
=2\end{array}$ & $\begin{array}{l}\alpha^{2} \\
=4\end{array}$ & $\begin{array}{c}\alpha^{3} \\
=\alpha+1 \\
=3\end{array}$ & $\begin{array}{c}\alpha^{4} \\
=\alpha^{2}+\alpha=6\end{array}$ & $\begin{array}{c}\alpha^{5} \\
=\alpha^{2}+\alpha+1=7\end{array}$ & $\begin{array}{c}\alpha^{6} \\
=\alpha^{2}+1 \\
=5\end{array}$ \\
\hline 0 & 0 & 0 & 0 & 0 & 0 & 0 & 0 & 0 \\
\hline $\begin{array}{c}\alpha^{7} \\
=1\end{array}$ & 0 & $\alpha^{7}=1$ & $\alpha=2$ & $\alpha^{2}=4$ & $\alpha^{3}=3$ & $\alpha^{4}=6$ & $\alpha^{5}=7$ & $\alpha^{6}=5$ \\
\hline $\begin{array}{c}\alpha \\
=2\end{array}$ & 0 & $\alpha=2$ & $\alpha^{2}=4$ & $\alpha^{3}=3$ & $\alpha^{4}=6$ & $\alpha^{5}=7$ & $\alpha^{6}=5$ & $\alpha^{7}=1$ \\
\hline $\begin{array}{l}\alpha^{2} \\
=4\end{array}$ & 0 & $\alpha^{2}=4$ & $\alpha^{3}=3$ & $\alpha^{4}=6$ & $\alpha^{5}=7$ & $\alpha^{6}=5$ & $\alpha^{7}=1$ & $\alpha=2$ \\
\hline $\begin{array}{c}\alpha^{3} \\
=\alpha+1 \\
=3\end{array}$ & 0 & $\alpha^{3}=3$ & $\alpha^{4}=6$ & $\alpha^{5}=7$ & $\alpha^{6}=5$ & $\alpha^{7}=1$ & $\alpha=2$ & $\alpha^{2}=4$ \\
\hline $\begin{array}{c}\alpha^{4} \\
=\alpha^{2}+\alpha \\
=6\end{array}$ & 0 & $\alpha^{4}=6$ & $\alpha^{5}=7$ & $\alpha^{6}=5$ & $\alpha^{7}=1$ & $\alpha=2$ & $\alpha^{2}=4$ & $\alpha^{3}=3$ \\
\hline $\begin{array}{c}\alpha^{5} \\
=\alpha^{2}+\alpha+1=7\end{array}$ & 0 & $\alpha^{5}=7$ & $\alpha^{6}=5$ & $\alpha^{7}=1$ & $\alpha=2$ & $\alpha^{2}=4$ & $\alpha^{3}=3$ & $\alpha^{4}=6$ \\
\hline $\begin{array}{c}\alpha^{6} \\
=\alpha^{2}+1 \\
=5\end{array}$ & 0 & $\alpha^{6}=5$ & $\alpha^{7}=1$ & $\alpha=2$ & $\alpha^{2}=4$ & $\alpha^{3}=3$ & $\alpha^{4}=6$ & $\alpha^{5}=7$ \\
\hline
\end{tabular}


Table 3 below presents the elements of GF(256)

\begin{tabular}{|c|c|c|c|c|c|}
\hline \multirow[b]{2}{*}{$\begin{array}{c}\text { Field } \\
\text { Element }\end{array}$} & \multirow[b]{2}{*}{$\begin{array}{c}\text { Alpha } \\
\text { Exponent }\end{array}$} & \multirow[b]{2}{*}{ Element Polynomial } & \multicolumn{2}{|c|}{ Exponent Values } & \multirow[b]{2}{*}{$\begin{array}{l}\text { Log of } \\
\text { Element }\end{array}$} \\
\hline & & & $\begin{array}{c}\text { Binary } \\
\text { Representation }\end{array}$ & $\begin{array}{c}\text { Decimal } \\
\text { Representation }\end{array}$ & \\
\hline 0 & (undefined) & 0 & 00000000 & 0 & (undefined) \\
\hline 1 & $\alpha^{0}$ & $\alpha^{0}$ & 00000001 & 1 & 0 \\
\hline 2 & $\alpha^{1}$ & $\alpha^{1}$ & 00000010 & 2 & 1 \\
\hline 3 & $\alpha^{2}$ & $\alpha^{2}$ & 00000100 & 4 & 25 \\
\hline 4 & $\alpha^{3}$ & $\alpha^{3}$ & 00001000 & 8 & . \\
\hline 5 & $\alpha^{4}$ & $\alpha^{4}$ & 00010000 & 16 & 50 \\
\hline 6 & $\alpha^{5}$ & $\alpha^{5}$ & 00100000 & 32 & 26 \\
\hline 7 & $\alpha^{6}$ & $\alpha^{6}$ & 01000000 & 64 & 198 \\
\hline 8 & $\alpha^{7}$ & $\alpha^{7}$ & 10000000 & 128 & 3 \\
\hline 9 & $\alpha^{8}$ & $\alpha^{4}+\alpha^{3}+\alpha^{2}+\alpha^{0}$ & 00011101 & 29 & 223 \\
\hline 10 & $\alpha^{9}$ & $\alpha^{5}+\alpha^{4}+\alpha^{3}+\alpha^{1}$ & 00111010 & 58 & 51 \\
\hline 11 & $\alpha^{10}$ & $\alpha^{6}+\alpha^{5}+\alpha^{4}+\alpha^{2}$ & 01110100 & 116 & 238 \\
\hline 12 & $\alpha^{11}$ & $\alpha^{7}+\alpha^{6}+\alpha^{5}+\alpha^{3}$ & 11101000 & 232 & 27 \\
\hline 13 & $\alpha^{12}$ & $\alpha^{7}+\alpha^{6}+\alpha^{3}+\alpha^{2}+\alpha^{0}$ & 11001101 & 205 & 104 \\
\hline 14 & $\alpha^{13}$ & $\alpha^{7}+\alpha^{2}+\alpha^{1}+\alpha^{0}$ & 10000111 & 135 & 199 \\
\hline 15 & $\alpha^{14}$ & $\alpha^{4}+\alpha^{1}+\alpha^{0}$ & 00010011 & 19 & 75 \\
\hline 16 & $\alpha^{15}$ & $\alpha^{5}+\alpha^{2}+\alpha^{1}$ & 00100110 & 38 & 4 \\
\hline 17 & $\alpha^{16}$ & $\alpha^{6}+\alpha^{3}+\alpha^{2}$ & 01001100 & 76 & 100 \\
\hline 18 & $\alpha^{17}$ & $\alpha^{7}+\alpha^{4}+\alpha^{3}$ & 10011000 & 152 & 224 \\
\hline 19 & $\alpha^{18}$ & $\alpha^{5}+\alpha^{3}+\alpha^{2}+\alpha^{0}$ & 00101101 & 45 & 14 \\
\hline 20 & $\alpha^{19}$ & $\alpha^{6}+\alpha^{4}+\alpha^{3}+\alpha^{1}$ & 01011010 & 90 & 52 \\
\hline 21 & $\alpha^{20}$ & $\alpha^{7}+\alpha^{5}+\alpha^{4}+\alpha^{2}$ & 10110100 & 180 & 141 \\
\hline 22 & $\alpha^{21}$ & $\alpha^{6}+\alpha^{5}+\alpha^{4}+\alpha^{2}+\alpha^{0}$ & 01110101 & 117 & 239 \\
\hline 23 & $\alpha^{22}$ & $\alpha^{7}+\alpha^{6}+\alpha^{5}+\alpha^{3}+\alpha^{1}$ & 11101010 & 234 & 129 \\
\hline 24 & $\alpha^{23}$ & $\alpha^{7}+\alpha^{6}+\alpha^{3}+\alpha^{0}$ & 11001001 & 201 & 28 \\
\hline 25 & $\alpha^{24}$ & $\alpha^{7}+\alpha^{3}+\alpha^{2}+\alpha^{1}+\alpha^{0}$ & 10001111 & 143 & 193 \\
\hline 26 & $\alpha^{25}$ & $\alpha^{1}+\alpha^{0}$ & 00000011 & 3 & 105 \\
\hline 27 & $\alpha^{26}$ & $\alpha^{2}+\alpha^{1}$ & 00000110 & 6 & 248 \\
\hline
\end{tabular}




\begin{tabular}{|c|c|c|c|c|c|}
\hline 28 & $\alpha^{27}$ & $\alpha^{3}+\alpha^{2}$ & 00001100 & 12 & 200 \\
\hline 29 & $\alpha^{28}$ & $\alpha^{4}+\alpha^{3}$ & 00011000 & 24 & 8 \\
\hline 30 & $\alpha^{29}$ & $\alpha^{5}+\alpha^{4}$ & 00110000 & 48 & 76 \\
\hline 31 & $\alpha^{30}$ & $\alpha^{6}+\alpha^{5}$ & 01100000 & 96 & 113 \\
\hline 32 & $\alpha^{31}$ & $\alpha^{7}+\alpha^{6}$ & 11000000 & 192 & 5 \\
\hline 33 & $\alpha^{32}$ & $\alpha^{7}+\alpha^{4}+\alpha^{3}+\alpha^{2}+\alpha^{0}$ & 10011101 & 157 & 138 \\
\hline 34 & $\alpha^{33}$ & $\alpha^{5}+\alpha^{2}+\alpha^{1}+\alpha^{0}$ & 00100111 & 39 & 101 \\
\hline 35 & $\alpha^{34}$ & $\alpha^{6}+\alpha^{3}+\alpha^{2}+\alpha^{1}$ & 01001110 & 78 & 47 \\
\hline 36 & $\alpha^{35}$ & $\alpha^{7}+\alpha^{4}+\alpha^{3}+\alpha^{2}$ & 10011100 & 156 & 225 \\
\hline 37 & $\alpha^{36}$ & $\alpha^{5}+\alpha^{2}+\alpha^{0}$ & 00100101 & 37 & 36 \\
\hline 38 & $\alpha^{37}$ & $\alpha^{6}+\alpha^{3}+\alpha^{1}$ & 01001010 & 74 & 15 \\
\hline 39 & $\alpha^{38}$ & $\alpha^{7}+\alpha^{4}+\alpha^{2}$ & 10010100 & 148 & 33 \\
\hline 40 & $\alpha^{39}$ & $\alpha^{5}+\alpha^{4}+\alpha^{2}+\alpha^{0}$ & 00110101 & 53 & 53 \\
\hline 41 & $\alpha^{40}$ & $\alpha^{6}+\alpha^{5}+\alpha^{3}+\alpha^{1}$ & 01101010 & 106 & 147 \\
\hline 42 & $\alpha^{41}$ & $\alpha^{7}+\alpha^{6}+\alpha^{4}+\alpha^{2}$ & 11010100 & 212 & 142 \\
\hline 43 & $\alpha^{42}$ & $\alpha^{7}+\alpha^{5}+\alpha^{4}+\alpha^{2}+\alpha^{0}$ & 10110101 & 181 & 218 \\
\hline 44 & $\alpha^{43}$ & $\alpha^{6}+\alpha^{5}+\alpha^{4}+\alpha^{2}+\alpha^{1}+\alpha^{0}$ & 01110111 & 119 & 240 \\
\hline 45 & $\alpha^{44}$ & $\alpha^{7}+\alpha^{6}+\alpha^{5}+\alpha^{3}+\alpha^{2}+\alpha^{1}$ & 11101110 & 238 & 18 \\
\hline 46 & $\alpha^{45}$ & $\alpha^{7}+\alpha^{6}+\alpha^{0}$ & 11000001 & 193 & 130 \\
\hline 47 & $\alpha^{46}$ & $\alpha^{7}+\alpha^{4}+\alpha^{3}+\alpha^{2}+\alpha^{1}+\alpha^{0}$ & 10011111 & 159 & 69 \\
\hline 48 & $\alpha^{47}$ & $\alpha^{5}+\alpha^{1}+\alpha^{0}$ & 00100011 & 35 & 29 \\
\hline 49 & $\alpha^{48}$ & $\alpha^{6}+\alpha^{2}+\alpha^{1}$ & 01000110 & 70 & 181 \\
\hline 50 & $\alpha^{49}$ & $\alpha^{7}+\alpha^{3}+\alpha^{2}$ & 10001100 & 140 & 194 \\
\hline 51 & $\alpha^{50}$ & $\alpha^{2}+\alpha^{0}$ & 00000101 & 5 & 125 \\
\hline 52 & $\alpha^{51}$ & $\alpha^{3}+\alpha^{1}$ & 00001010 & 10 & 106 \\
\hline 53 & $\alpha^{52}$ & $\alpha^{4}+\alpha^{2}$ & 00010100 & 20 & 39 \\
\hline 54 & $\alpha^{53}$ & $\alpha^{5}+\alpha^{3}$ & 00101000 & 40 & 249 \\
\hline 55 & $\alpha^{54}$ & $\alpha^{6}+\alpha^{4}$ & 01010000 & 80 & 185 \\
\hline 56 & $\alpha^{55}$ & $\alpha^{7}+\alpha^{5}$ & 10100000 & 160 & 201 \\
\hline 57 & $\alpha^{56}$ & $\alpha^{6}+\alpha^{4}+\alpha^{3}+\alpha^{2}+\alpha^{0}$ & 01011101 & 93 & 154 \\
\hline 58 & $\alpha^{57}$ & $\alpha^{7}+\alpha^{5}+\alpha^{4}+\alpha^{3}+\alpha^{1}$ & 10111010 & 186 & 9 \\
\hline 59 & $\alpha^{58}$ & $\alpha^{6}+\alpha^{5}+\alpha^{3}+\alpha^{0}$ & 01101001 & 105 & 120 \\
\hline 60 & $\alpha^{59}$ & $\alpha^{7}+\alpha^{6}+\alpha^{4}+\alpha^{1}$ & 11010010 & 210 & 77 \\
\hline 61 & $\alpha^{60}$ & $\alpha^{7}+\alpha^{5}+\alpha^{4}+\alpha^{3}+\alpha^{0}$ & 10111001 & 185 & 228 \\
\hline
\end{tabular}




\begin{tabular}{|c|c|c|c|c|c|}
\hline 62 & $\alpha^{61}$ & $\alpha^{6}+\alpha^{5}+\alpha^{3}+\alpha^{2}+\alpha^{1}+\alpha^{0}$ & 01101111 & 111 & 114 \\
\hline 63 & $\alpha^{62}$ & $\alpha^{7}+\alpha^{6}+\alpha^{4}+\alpha^{3}+\alpha^{2}+\alpha^{1}$ & 11011110 & 222 & 166 \\
\hline 64 & $\alpha^{63}$ & $\alpha^{7}+\alpha^{5}+\alpha^{0}$ & 10100001 & 161 & 6 \\
\hline 65 & $\alpha^{64}$ & $\alpha^{6}+\alpha^{4}+\alpha^{3}+\alpha^{2}+\alpha^{1}+\alpha^{0}$ & 01011111 & 95 & 191 \\
\hline 66 & $\alpha^{65}$ & $\alpha^{7}+\alpha^{5}+\alpha^{4}+\alpha^{3}+\alpha^{2}+\alpha^{1}$ & 10111110 & 190 & 139 \\
\hline 67 & $\alpha^{66}$ & $\alpha^{6}+\alpha^{5}+\alpha^{0}$ & 01100001 & 97 & 98 \\
\hline 68 & $\alpha^{67}$ & $\alpha^{7}+\alpha^{6}+\alpha^{1}$ & 11000010 & 194 & 102 \\
\hline 69 & $\alpha^{68}$ & $\alpha^{7}+\alpha^{4}+\alpha^{3}+\alpha^{0}$ & 10011001 & 153 & 221 \\
\hline 70 & $\alpha^{69}$ & $\alpha^{5}+\alpha^{3}+\alpha^{2}+\alpha^{1}+\alpha^{0}$ & 00101111 & 47 & 48 \\
\hline 71 & $\alpha^{70}$ & $\alpha^{6}+\alpha^{4}+\alpha^{3}+\alpha^{2}+\alpha^{1}$ & 01011110 & 94 & 253 \\
\hline 72 & $\alpha^{71}$ & $\alpha^{7}+\alpha^{5}+\alpha^{4}+\alpha^{3}+\alpha^{2}$ & 10111100 & 188 & 226 \\
\hline 73 & $\alpha^{72}$ & $\alpha^{6}+\alpha^{5}+\alpha^{2}+\alpha^{0}$ & 01100101 & 101 & 152 \\
\hline 74 & $\alpha^{73}$ & $\alpha^{7}+\alpha^{6}+\alpha^{3}+\alpha^{1}$ & 11001010 & 202 & 37 \\
\hline 75 & $\alpha^{74}$ & $\alpha^{7}+\alpha^{3}+\alpha^{0}$ & 10001001 & 137 & 179 \\
\hline 76 & $\alpha^{75}$ & $\alpha^{3}+\alpha^{2}+\alpha^{1}+\alpha^{0}$ & 00001111 & 15 & 16 \\
\hline 77 & $\alpha^{76}$ & $\alpha^{4}+\alpha^{3}+\alpha^{2}+\alpha^{1}$ & 00011110 & 30 & 145 \\
\hline 78 & $\alpha^{77}$ & $\alpha^{5}+\alpha^{4}+\alpha^{3}+\alpha^{2}$ & 00111100 & 60 & 34 \\
\hline 79 & $\alpha^{78}$ & $\alpha^{6}+\alpha^{5}+\alpha^{4}+\alpha^{3}$ & 01111000 & 120 & 136 \\
\hline 80 & $\alpha^{79}$ & $\alpha^{7}+\alpha^{6}+\alpha^{5}+\alpha^{4}$ & 11110000 & 240 & 54 \\
\hline 81 & $\alpha^{80}$ & $\alpha^{7}+\alpha^{6}+\alpha^{5}+\alpha^{4}+\alpha^{3}+\alpha^{2}+\alpha^{0}$ & 11111101 & 253 & 208 \\
\hline 82 & $\alpha^{81}$ & $\alpha^{7}+\alpha^{6}+\alpha^{5}+\alpha^{2}+\alpha^{1}+\alpha^{0}$ & 11100111 & 231 & 148 \\
\hline 83 & $\alpha^{82}$ & $\alpha^{7}+\alpha^{6}+\alpha^{4}+\alpha^{1}+\alpha^{0}$ & 11010011 & 211 & 206 \\
\hline 84 & $\alpha^{83}$ & $\alpha^{7}+\alpha^{5}+\alpha^{4}+\alpha^{3}+\alpha^{1}+\alpha^{0}$ & 10111011 & 187 & 143 \\
\hline 85 & $\alpha^{84}$ & $\alpha^{6}+\alpha^{5}+\alpha^{3}+\alpha^{1}+\alpha^{0}$ & 01101011 & 107 & 150 \\
\hline 86 & $\alpha^{85}$ & $\alpha^{7}+\alpha^{6}+\alpha^{4}+\alpha^{2}+\alpha^{1}$ & 11010110 & 214 & 219 \\
\hline 87 & $\alpha^{86}$ & $\alpha^{7}+\alpha^{5}+\alpha^{4}+\alpha^{0}$ & 10110001 & 177 & 189 \\
\hline 88 & $\alpha^{87}$ & $\alpha^{6}+\alpha^{5}+\alpha^{4}+\alpha^{3}+\alpha^{2}+\alpha^{1}+\alpha^{0}$ & 01111111 & 127 & 241 \\
\hline 89 & $\alpha^{88}$ & $\alpha^{7}+\alpha^{6}+\alpha^{5}+\alpha^{4}+\alpha^{3}+\alpha^{2}+\alpha^{1}$ & 11111110 & 254 & 210 \\
\hline 90 & $\alpha^{89}$ & $\alpha^{7}+\alpha^{6}+\alpha^{5}+\alpha^{0}$ & 11100001 & 225 & 19 \\
\hline 91 & $\alpha^{90}$ & $\alpha^{7}+\alpha^{6}+\alpha^{4}+\alpha^{3}+\alpha^{2}+\alpha^{1}+\alpha^{0}$ & 11011111 & 223 & 92 \\
\hline 92 & $\alpha^{91}$ & $\alpha^{7}+\alpha^{5}+\alpha^{1}+\alpha^{0}$ & 10100011 & 163 & 131 \\
\hline 93 & $\alpha^{92}$ & $\alpha^{6}+\alpha^{4}+\alpha^{3}+\alpha^{1}+\alpha^{0}$ & 01011011 & 91 & 56 \\
\hline
\end{tabular}




\begin{tabular}{|c|c|c|c|c|c|}
\hline 94 & $\alpha^{93}$ & $\alpha^{7}+\alpha^{5}+\alpha^{4}+\alpha^{2}+\alpha^{1}$ & 10110110 & 182 & 70 \\
\hline 95 & $\alpha^{94}$ & $\alpha^{6}+\alpha^{5}+\alpha^{4}+\alpha^{0}$ & 01110001 & 113 & 64 \\
\hline 96 & $\alpha^{95}$ & $\alpha^{7}+\alpha^{6}+\alpha^{5}+\alpha^{1}$ & 11100010 & 226 & 30 \\
\hline 97 & $\alpha^{96}$ & $\alpha^{7}+\alpha^{6}+\alpha^{4}+\alpha^{3}+\alpha^{0}$ & 11011001 & 217 & 66 \\
\hline 98 & $\alpha^{97}$ & $\alpha^{7}+\alpha^{5}+\alpha^{3}+\alpha^{2}+\alpha^{1}+\alpha^{0}$ & 10101111 & 175 & 182 \\
\hline 99 & $\alpha^{98}$ & $\alpha^{6}+\alpha^{1}+\alpha^{0}$ & 01000011 & 67 & 163 \\
\hline 100 & $\alpha^{99}$ & $\alpha^{7}+\alpha^{2}+\alpha^{1}$ & 10000110 & 134 & 195 \\
\hline 101 & $\alpha^{100}$ & $\alpha^{4}+\alpha^{0}$ & 00010001 & 17 & 72 \\
\hline 102 & $\alpha^{101}$ & $\alpha^{5}+\alpha^{1}$ & 00100010 & 34 & 126 \\
\hline 103 & $\alpha^{102}$ & $\alpha^{6}+\alpha^{2}$ & 01000100 & 68 & 110 \\
\hline 104 & $\alpha^{103}$ & $\alpha^{7}+\alpha^{3}$ & 10001000 & 136 & 107 \\
\hline 105 & $\alpha^{104}$ & $\alpha^{3}+\alpha^{2}+\alpha^{0}$ & 00001101 & 13 & 58 \\
\hline 106 & $\alpha^{105}$ & $\alpha^{4}+\alpha^{3}+\alpha^{1}$ & 00011010 & 26 & 40 \\
\hline 107 & $\alpha^{106}$ & $\alpha^{5}+\alpha^{4}+\alpha^{2}$ & 00110100 & 52 & 84 \\
\hline 108 & $\alpha^{107}$ & $\alpha^{6}+\alpha^{5}+\alpha^{3}$ & 01101000 & 104 & 250 \\
\hline 109 & $\alpha^{108}$ & $\alpha^{7}+\alpha^{6}+\alpha^{4}$ & 11010000 & 208 & 133 \\
\hline 110 & $\alpha^{109}$ & $\alpha^{7}+\alpha^{5}+\alpha^{4}+\alpha^{3}+\alpha^{2}+\alpha^{0}$ & 10111101 & 189 & 186 \\
\hline 111 & $\alpha^{110}$ & $\alpha^{6}+\alpha^{5}+\alpha^{2}+\alpha^{1}+\alpha^{0}$ & 01100111 & 103 & 61 \\
\hline 112 & $\alpha^{111}$ & $\alpha^{7}+\alpha^{6}+\alpha^{3}+\alpha^{2}+\alpha^{1}$ & 11001110 & 206 & 202 \\
\hline 113 & $\alpha^{112}$ & $\alpha^{7}+\alpha^{0}$ & 10000001 & 129 & 94 \\
\hline 114 & $\alpha^{113}$ & $\alpha^{4}+\alpha^{3}+\alpha^{2}+\alpha^{1}+\alpha^{0}$ & 00011111 & 31 & 155 \\
\hline 115 & $\alpha^{114}$ & $\alpha^{5}+\alpha^{4}+\alpha^{3}+\alpha^{2}+\alpha^{1}$ & 00111110 & 62 & 159 \\
\hline 116 & $\alpha^{115}$ & $\alpha^{6}+\alpha^{5}+\alpha^{4}+\alpha^{3}+\alpha^{2}$ & 01111100 & 124 & 10 \\
\hline 117 & $\alpha^{116}$ & $\alpha^{7}+\alpha^{6}+\alpha^{5}+\alpha^{4}+\alpha^{3}$ & 11111000 & 248 & 21 \\
\hline 118 & $\alpha^{117}$ & $\alpha^{7}+\alpha^{6}+\alpha^{5}+\alpha^{3}+\alpha^{2}+\alpha^{0}$ & 11101101 & 237 & 121 \\
\hline 119 & $\alpha^{118}$ & $\alpha^{7}+\alpha^{6}+\alpha^{2}+\alpha^{1}+\alpha^{0}$ & 11000111 & 199 & 43 \\
\hline 120 & $\alpha^{119}$ & $\alpha^{7}+\alpha^{4}+\alpha^{1}+\alpha^{0}$ & 10010011 & 147 & 78 \\
\hline 121 & $\alpha^{120}$ & $\alpha^{5}+\alpha^{4}+\alpha^{3}+\alpha^{1}+\alpha^{0}$ & 00111011 & 59 & 212 \\
\hline 122 & $\alpha^{121}$ & $\alpha^{6}+\alpha^{5}+\alpha^{4}+\alpha^{2}+\alpha^{1}$ & 01110110 & 118 & 229 \\
\hline 123 & $\alpha^{122}$ & $\alpha^{7}+\alpha^{6}+\alpha^{5}+\alpha^{3}+\alpha^{2}$ & 11101100 & 236 & 172 \\
\hline 124 & $\alpha^{123}$ & $\alpha^{7}+\alpha^{6}+\alpha^{2}+\alpha^{0}$ & 11000101 & 197 & 115 \\
\hline 125 & $\alpha^{124}$ & $\alpha^{7}+\alpha^{4}+\alpha^{2}+\alpha^{1}+\alpha^{0}$ & 10010111 & 151 & 243 \\
\hline
\end{tabular}




\begin{tabular}{|c|c|c|c|c|c|}
\hline 126 & $\alpha^{125}$ & $\alpha^{5}+\alpha^{4}+\alpha^{1}+\alpha^{0}$ & 00110011 & 51 & 167 \\
\hline 127 & $\alpha^{126}$ & $\alpha^{6}+\alpha^{5}+\alpha^{2}+\alpha^{1}$ & 01100110 & 102 & 87 \\
\hline 128 & $\alpha^{127}$ & $\alpha^{7}+\alpha^{6}+\alpha^{3}+\alpha^{2}$ & 11001100 & 204 & 7 \\
\hline 129 & $\alpha^{128}$ & $\alpha^{7}+\alpha^{2}+\alpha^{0}$ & 10000101 & 133 & 112 \\
\hline 130 & $\alpha^{129}$ & $\alpha^{4}+\alpha^{2}+\alpha^{1}+\alpha^{0}$ & 00010111 & 23 & 192 \\
\hline 131 & $\alpha^{130}$ & $\alpha^{5}+\alpha^{3}+\alpha^{2}+\alpha^{1}$ & 00101110 & 46 & 247 \\
\hline 132 & $\alpha^{131}$ & $\alpha^{6}+\alpha^{4}+\alpha^{3}+\alpha^{2}$ & 01011100 & 92 & 140 \\
\hline 133 & $\alpha^{132}$ & $\alpha^{7}+\alpha^{5}+\alpha^{4}+\alpha^{3}$ & 10111000 & 184 & 128 \\
\hline 134 & $\alpha^{133}$ & $\alpha^{6}+\alpha^{5}+\alpha^{3}+\alpha^{2}+\alpha^{0}$ & 01101101 & 109 & 99 \\
\hline 135 & $\alpha^{134}$ & $\alpha^{7}+\alpha^{6}+\alpha^{4}+\alpha^{3}+\alpha^{1}$ & 11011010 & 218 & 13 \\
\hline 136 & $\alpha^{135}$ & $\alpha^{7}+\alpha^{5}+\alpha^{3}+\alpha^{0}$ & 10101001 & 169 & 103 \\
\hline 137 & $\alpha^{136}$ & $\alpha^{6}+\alpha^{3}+\alpha^{2}+\alpha^{1}+\alpha^{0}$ & 01001111 & 79 & 74 \\
\hline 138 & $\alpha^{137}$ & $\alpha^{7}+\alpha^{4}+\alpha^{3}+\alpha^{2}+\alpha^{1}$ & 10011110 & 158 & 222 \\
\hline 139 & $\alpha^{138}$ & $\alpha^{5}+\alpha^{0}$ & 00100001 & 33 & 237 \\
\hline 140 & $\alpha^{139}$ & $\alpha^{6}+\alpha^{1}$ & 01000010 & 66 & 49 \\
\hline 141 & $\alpha^{140}$ & $\alpha^{7}+\alpha^{2}$ & 10000100 & 132 & 197 \\
\hline 142 & $\alpha^{141}$ & $\alpha^{4}+\alpha^{2}+\alpha^{0}$ & 00010101 & 21 & 254 \\
\hline 143 & $\alpha^{142}$ & $\alpha^{5}+\alpha^{3}+\alpha^{1}$ & 00101010 & 42 & 24 \\
\hline 144 & $\alpha^{143}$ & $\alpha^{6}+\alpha^{4}+\alpha^{2}$ & 01010100 & 84 & 227 \\
\hline 145 & $\alpha^{144}$ & $\alpha^{7}+\alpha^{5}+\alpha^{3}$ & 10101000 & 168 & 165 \\
\hline 146 & $\alpha^{145}$ & $\alpha^{6}+\alpha^{3}+\alpha^{2}+\alpha^{0}$ & 01001101 & 77 & 153 \\
\hline 147 & $\alpha^{146}$ & $\alpha^{7}+\alpha^{4}+\alpha^{3}+\alpha^{1}$ & 10011010 & 154 & 119 \\
\hline 148 & $\alpha^{147}$ & $\alpha^{5}+\alpha^{3}+\alpha^{0}$ & 00101001 & 41 & 38 \\
\hline 149 & $\alpha^{148}$ & $\alpha^{6}+\alpha^{4}+\alpha^{1}$ & 01010010 & 82 & 184 \\
\hline 150 & $\alpha^{149}$ & $\alpha^{7}+\alpha^{5}+\alpha^{2}$ & 10100100 & 164 & 180 \\
\hline 151 & $\alpha^{150}$ & $\alpha^{6}+\alpha^{4}+\alpha^{2}+\alpha^{0}$ & 01010101 & 85 & 124 \\
\hline 152 & $\alpha^{151}$ & $\alpha^{7}+\alpha^{5}+\alpha^{3}+\alpha^{1}$ & 10101010 & 170 & 17 \\
\hline 153 & $\alpha^{152}$ & $\alpha^{6}+\alpha^{3}+\alpha^{0}$ & 01001001 & 73 & 68 \\
\hline 154 & $\alpha^{153}$ & $\alpha^{7}+\alpha^{4}+\alpha^{1}$ & 10010010 & 146 & 146 \\
\hline 155 & $\alpha^{154}$ & $\alpha^{5}+\alpha^{4}+\alpha^{3}+\alpha^{0}$ & 00111001 & 57 & 217 \\
\hline 156 & $\alpha^{155}$ & $\alpha^{6}+\alpha^{5}+\alpha^{4}+\alpha^{1}$ & 01110010 & 114 & 35 \\
\hline 157 & $\alpha^{156}$ & $\alpha^{7}+\alpha^{6}+\alpha^{5}+\alpha^{2}$ & 11100100 & 228 & 32 \\
\hline
\end{tabular}




\begin{tabular}{|c|c|c|c|c|c|}
\hline 158 & $\alpha^{157}$ & $\alpha^{7}+\alpha^{6}+\alpha^{4}+\alpha^{2}+\alpha^{0}$ & 11010101 & 213 & 137 \\
\hline 159 & $\alpha^{158}$ & $\alpha^{7}+\alpha^{5}+\alpha^{4}+\alpha^{2}+\alpha^{1}+\alpha^{0}$ & 10110111 & 183 & 46 \\
\hline 160 & $\alpha^{159}$ & $\alpha^{6}+\alpha^{5}+\alpha^{4}+\alpha^{1}+\alpha^{0}$ & 01110011 & 115 & 55 \\
\hline 161 & $\alpha^{160}$ & $\alpha^{7}+\alpha^{6}+\alpha^{5}+\alpha^{2}+\alpha^{1}$ & 11100110 & 230 & 63 \\
\hline 162 & $\alpha^{161}$ & $\alpha^{7}+\alpha^{6}+\alpha^{4}+\alpha^{0}$ & 11010001 & 209 & 209 \\
\hline 163 & $\alpha^{162}$ & $\alpha^{7}+\alpha^{5}+\alpha^{4}+\alpha^{3}+\alpha^{2}+\alpha^{1}+\alpha^{0}$ & 10111111 & 191 & 91 \\
\hline 164 & $\alpha^{163}$ & $\alpha^{6}+\alpha^{5}+\alpha^{1}+\alpha^{0}$ & 01100011 & 99 & 149 \\
\hline 165 & $\alpha^{164}$ & $\alpha^{7}+\alpha^{6}+\alpha^{2}+\alpha^{1}$ & 11000110 & 198 & 188 \\
\hline 166 & $\alpha^{165}$ & $\alpha^{7}+\alpha^{4}+\alpha^{0}$ & 10010001 & 145 & 207 \\
\hline 167 & $\alpha^{166}$ & $\alpha^{5}+\alpha^{4}+\alpha^{3}+\alpha^{2}+\alpha^{1}+\alpha^{0}$ & 00111111 & 63 & 205 \\
\hline 168 & $\alpha^{167}$ & $\alpha^{6}+\alpha^{5}+\alpha^{4}+\alpha^{3}+\alpha^{2}+\alpha^{1}$ & 01111110 & 126 & 144 \\
\hline 169 & $\alpha^{168}$ & $\alpha^{7}+\alpha^{6}+\alpha^{5}+\alpha^{4}+\alpha^{3}+\alpha^{2}$ & 11111100 & 252 & 135 \\
\hline 170 & $\alpha^{169}$ & $\alpha^{7}+\alpha^{6}+\alpha^{5}+\alpha^{2}+\alpha^{0}$ & 11100101 & 229 & 151 \\
\hline 171 & $\alpha^{170}$ & $\alpha^{7}+\alpha^{6}+\alpha^{4}+\alpha^{2}+\alpha^{1}+\alpha^{0}$ & 11010111 & 215 & 178 \\
\hline 172 & $\alpha^{171}$ & $\alpha^{7}+\alpha^{5}+\alpha^{4}+\alpha^{1}+\alpha^{0}$ & 10110011 & 179 & 220 \\
\hline 173 & $\alpha^{172}$ & $\alpha^{6}+\alpha^{5}+\alpha^{4}+\alpha^{3}+\alpha^{1}+\alpha^{0}$ & 01111011 & 123 & 252 \\
\hline 174 & $\alpha^{173}$ & $\alpha^{7}+\alpha^{6}+\alpha^{5}+\alpha^{4}+\alpha^{2}+\alpha^{1}$ & 11110110 & 246 & 190 \\
\hline 175 & $\alpha^{174}$ & $\alpha^{7}+\alpha^{6}+\alpha^{5}+\alpha^{4}+\alpha^{0}$ & 11110001 & 241 & 97 \\
\hline 176 & $\alpha^{175}$ & $\alpha^{7}+\alpha^{6}+\alpha^{5}+\alpha^{4}+\alpha^{3}+\alpha^{2}+\alpha^{1}+\alpha^{0}$ & 11111111 & 255 & 242 \\
\hline 177 & $\alpha^{176}$ & $\alpha^{7}+\alpha^{6}+\alpha^{5}+\alpha^{1}+\alpha^{0}$ & 11100011 & 227 & 86 \\
\hline 178 & $\alpha^{177}$ & $\alpha^{7}+\alpha^{6}+\alpha^{4}+\alpha^{3}+\alpha^{1}+\alpha^{0}$ & 11011011 & 219 & 211 \\
\hline 179 & $\alpha^{178}$ & $\alpha^{7}+\alpha^{5}+\alpha^{3}+\alpha^{1}+\alpha^{0}$ & 10101011 & 171 & 171 \\
\hline 180 & $\alpha^{179}$ & $\alpha^{6}+\alpha^{3}+\alpha^{1}+\alpha^{0}$ & 01001011 & 75 & 20 \\
\hline 181 & $\alpha^{180}$ & $\alpha^{7}+\alpha^{4}+\alpha^{2}+\alpha^{1}$ & 10010110 & 150 & 42 \\
\hline 182 & $\alpha^{181}$ & $\alpha^{5}+\alpha^{4}+\alpha^{0}$ & 00110001 & 49 & 93 \\
\hline 183 & $\alpha^{182}$ & $\alpha^{6}+\alpha^{5}+\alpha^{1}$ & 01100010 & 98 & 158 \\
\hline 184 & $\alpha^{183}$ & $\alpha^{7}+\alpha^{6}+\alpha^{2}$ & 11000100 & 196 & 132 \\
\hline 185 & $\alpha^{184}$ & $\alpha^{7}+\alpha^{4}+\alpha^{2}+\alpha^{0}$ & 10010101 & 149 & 60 \\
\hline 186 & $\alpha^{185}$ & $\alpha^{5}+\alpha^{4}+\alpha^{2}+\alpha^{1}+\alpha^{0}$ & 00110111 & 55 & 57 \\
\hline 187 & $\alpha^{186}$ & $\alpha^{6}+\alpha^{5}+\alpha^{3}+\alpha^{2}+\alpha^{1}$ & 01101110 & 110 & 83 \\
\hline 188 & $\alpha^{187}$ & $\alpha^{7}+\alpha^{6}+\alpha^{4}+\alpha^{3}+\alpha^{2}$ & 11011100 & 220 & 71 \\
\hline 189 & $\alpha^{188}$ & $\alpha^{7}+\alpha^{5}+\alpha^{2}+\alpha^{0}$ & 10100101 & 165 & 109 \\
\hline
\end{tabular}




\begin{tabular}{|c|c|c|c|c|c|}
\hline 190 & $\alpha^{189}$ & $\alpha^{6}+\alpha^{4}+\alpha^{2}+\alpha^{1}+\alpha^{0}$ & 01010111 & 87 & 65 \\
\hline 191 & $\alpha^{190}$ & $\alpha^{7}+\alpha^{5}+\alpha^{3}+\alpha^{2}+\alpha^{1}$ & 10101110 & 174 & 162 \\
\hline 192 & $\alpha^{191}$ & $\alpha^{6}+\alpha^{0}$ & 01000001 & 65 & 31 \\
\hline 193 & $\alpha^{192}$ & $\alpha^{7}+\alpha^{1}$ & 10000010 & 130 & 45 \\
\hline 194 & $\alpha^{193}$ & $\alpha^{4}+\alpha^{3}+\alpha^{0}$ & 00011001 & 25 & 67 \\
\hline 195 & $\alpha^{194}$ & $\alpha^{5}+\alpha^{4}+\alpha^{1}$ & 00110010 & 50 & 216 \\
\hline 196 & $\alpha^{195}$ & $\alpha^{6}+\alpha^{5}+\alpha^{2}$ & 01100100 & 100 & 183 \\
\hline 197 & $\alpha^{196}$ & $\alpha^{7}+\alpha^{6}+\alpha^{3}$ & 11001000 & 200 & 123 \\
\hline 198 & $\alpha^{197}$ & $\alpha^{7}+\alpha^{3}+\alpha^{2}+\alpha^{0}$ & 10001101 & 141 & 164 \\
\hline 199 & $\alpha^{198}$ & $\alpha^{2}+\alpha^{1}+\alpha^{0}$ & 00000111 & 7 & 118 \\
\hline 200 & $\alpha^{199}$ & $\alpha^{3}+\alpha^{2}+\alpha^{1}$ & 00001110 & 14 & 196 \\
\hline 201 & $\alpha^{200}$ & $\alpha^{4}+\alpha^{3}+\alpha^{2}$ & 00011100 & 28 & 23 \\
\hline 202 & $\alpha^{201}$ & $\alpha^{5}+\alpha^{4}+\alpha^{3}$ & 00111000 & 56 & 73 \\
\hline 203 & $\alpha^{202}$ & $\alpha^{6}+\alpha^{5}+\alpha^{4}$ & 01110000 & 112 & 236 \\
\hline 204 & $\alpha^{203}$ & $\alpha^{7}+\alpha^{6}+\alpha^{5}$ & 11100000 & 224 & 127 \\
\hline 205 & $\alpha^{204}$ & $\alpha^{7}+\alpha^{6}+\alpha^{4}+\alpha^{3}+\alpha^{2}+\alpha^{0}$ & 11011101 & 221 & 12 \\
\hline 206 & $\alpha^{205}$ & $\alpha^{7}+\alpha^{5}+\alpha^{2}+\alpha^{1}+\alpha^{0}$ & 10100111 & 167 & 111 \\
\hline 207 & $\alpha^{206}$ & $\alpha^{6}+\alpha^{4}+\alpha^{1}+\alpha^{0}$ & 01010011 & 83 & 246 \\
\hline 208 & $\alpha^{207}$ & $\alpha^{7}+\alpha^{5}+\alpha^{2}+\alpha^{1}$ & 10100110 & 166 & 108 \\
\hline 209 & $\alpha^{208}$ & $\alpha^{6}+\alpha^{4}+\alpha^{0}$ & 01010001 & 81 & 161 \\
\hline 210 & $\alpha^{209}$ & $\alpha^{7}+\alpha^{5}+\alpha^{1}$ & 10100010 & 162 & 59 \\
\hline 211 & $\alpha^{210}$ & $\alpha^{6}+\alpha^{4}+\alpha^{3}+\alpha^{0}$ & 01011001 & 89 & 82 \\
\hline 212 & $\alpha^{211}$ & $\alpha^{7}+\alpha^{5}+\alpha^{4}+\alpha^{1}$ & 10110010 & 178 & 41 \\
\hline 213 & $\alpha^{212}$ & $\alpha^{6}+\alpha^{5}+\alpha^{4}+\alpha^{3}+\alpha^{0}$ & 01111001 & 121 & 157 \\
\hline 214 & $\alpha^{213}$ & $\alpha^{7}+\alpha^{6}+\alpha^{5}+\alpha^{4}+\alpha^{1}$ & 11110010 & 242 & 85 \\
\hline 215 & $\alpha^{214}$ & $\alpha^{7}+\alpha^{6}+\alpha^{5}+\alpha^{4}+\alpha^{3}+\alpha^{0}$ & 11111001 & 249 & 170 \\
\hline 216 & $\alpha^{215}$ & $\alpha^{7}+\alpha^{6}+\alpha^{5}+\alpha^{3}+\alpha^{2}+\alpha^{1}+\alpha^{0}$ & 11101111 & 239 & 251 \\
\hline 217 & $\alpha^{216}$ & $\alpha^{7}+\alpha^{6}+\alpha^{1}+\alpha^{0}$ & 11000011 & 195 & 96 \\
\hline 218 & $\alpha^{217}$ & $\alpha^{7}+\alpha^{4}+\alpha^{3}+\alpha^{1}+\alpha^{0}$ & 10011011 & 155 & 134 \\
\hline 219 & $\alpha^{218}$ & $\alpha^{5}+\alpha^{3}+\alpha^{1}+\alpha^{0}$ & 00101011 & 43 & 177 \\
\hline 220 & $\alpha^{219}$ & $\alpha^{6}+\alpha^{4}+\alpha^{2}+\alpha^{1}$ & 01010110 & 86 & 187 \\
\hline
\end{tabular}




\begin{tabular}{|c|c|c|c|c|c|}
\hline 221 & $\alpha^{220}$ & $\alpha^{7}+\alpha^{5}+\alpha^{3}+\alpha^{2}$ & 10101100 & 172 & 204 \\
\hline 222 & $\alpha^{221}$ & $\alpha^{6}+\alpha^{2}+\alpha^{0}$ & 01000101 & 69 & 62 \\
\hline 223 & $\alpha^{222}$ & $\alpha^{7}+\alpha^{3}+\alpha^{1}$ & 10001010 & 138 & 90 \\
\hline 224 & $\alpha^{223}$ & $\alpha^{3}+\alpha^{0}$ & 00001001 & 9 & 203 \\
\hline 225 & $\alpha^{224}$ & $\alpha^{4}+\alpha^{1}$ & 00010010 & 18 & 89 \\
\hline 226 & $\alpha^{225}$ & $\alpha^{5}+\alpha^{2}$ & 00100100 & 36 & 95 \\
\hline 227 & $\alpha^{226}$ & $\alpha^{6}+\alpha^{3}$ & 01001000 & 72 & 176 \\
\hline 228 & $\alpha^{227}$ & $\alpha^{7}+\alpha^{4}$ & 10010000 & 144 & 156 \\
\hline 229 & $\alpha^{228}$ & $\alpha^{5}+\alpha^{4}+\alpha^{3}+\alpha^{2}+\alpha^{0}$ & 00111101 & 61 & 169 \\
\hline 230 & $\alpha^{229}$ & $\alpha^{6}+\alpha^{5}+\alpha^{4}+\alpha^{3}+\alpha^{1}$ & 01111010 & 122 & 160 \\
\hline 231 & $\alpha^{230}$ & $\alpha^{7}+\alpha^{6}+\alpha^{5}+\alpha^{4}+\alpha^{2}$ & 11110100 & 244 & 81 \\
\hline 232 & $\alpha^{231}$ & $\alpha^{7}+\alpha^{6}+\alpha^{5}+\alpha^{4}+\alpha^{2}+\alpha^{0}$ & 11110101 & 245 & 11 \\
\hline 233 & $\alpha^{232}$ & $\alpha^{7}+\alpha^{6}+\alpha^{5}+\alpha^{4}+\alpha^{2}+\alpha^{1}+\alpha^{0}$ & 11110111 & 247 & 245 \\
\hline 234 & $\alpha^{233}$ & $\alpha^{7}+\alpha^{6}+\alpha^{5}+\alpha^{4}+\alpha^{1}+\alpha^{0}$ & 11110011 & 243 & 22 \\
\hline 235 & $\alpha^{234}$ & $\alpha^{7}+\alpha^{6}+\alpha^{5}+\alpha^{4}+\alpha^{3}+\alpha^{1}+\alpha^{0}$ & 11111011 & 251 & 235 \\
\hline 236 & $\alpha^{235}$ & $\alpha^{7}+\alpha^{6}+\alpha^{5}+\alpha^{3}+\alpha^{1}+\alpha^{0}$ & 11101011 & 235 & 122 \\
\hline 237 & $\alpha^{236}$ & $\alpha^{7}+\alpha^{6}+\alpha^{3}+\alpha^{1}+\alpha^{0}$ & 11001011 & 203 & 117 \\
\hline 238 & $\alpha^{237}$ & $\alpha^{7}+\alpha^{3}+\alpha^{1}+\alpha^{0}$ & 10001011 & 139 & 44 \\
\hline 239 & $\alpha^{238}$ & $\alpha^{3}+\alpha^{1}+\alpha^{0}$ & 00001011 & 11 & 215 \\
\hline 240 & $\alpha^{239}$ & $\alpha^{4}+\alpha^{2}+\alpha^{1}$ & 00010110 & 22 & 79 \\
\hline 241 & $\alpha^{240}$ & $\alpha^{5}+\alpha^{3}+\alpha^{2}$ & 00101100 & 44 & 174 \\
\hline 242 & $\alpha^{241}$ & $\alpha^{6}+\alpha^{4}+\alpha^{3}$ & 01011000 & 88 & 213 \\
\hline 243 & $\alpha^{242}$ & $\alpha^{7}+\alpha^{5}+\alpha^{4}$ & 10110000 & 176 & 233 \\
\hline 244 & $\alpha^{243}$ & $\alpha^{6}+\alpha^{5}+\alpha^{4}+\alpha^{3}+\alpha^{2}+\alpha^{0}$ & 01111101 & 125 & 230 \\
\hline 245 & $\alpha^{244}$ & $\alpha^{7}+\alpha^{6}+\alpha^{5}+\alpha^{4}+\alpha^{3}+\alpha^{1}$ & 11111010 & 250 & 231 \\
\hline 246 & $\alpha^{245}$ & $\alpha^{7}+\alpha^{6}+\alpha^{5}+\alpha^{3}+\alpha^{0}$ & 11101001 & 233 & 173 \\
\hline 247 & $\alpha^{246}$ & $\alpha^{7}+\alpha^{6}+\alpha^{3}+\alpha^{2}+\alpha^{1}+\alpha^{0}$ & 11001111 & 207 & 232 \\
\hline 248 & $\alpha^{247}$ & $\alpha^{7}+\alpha^{1}+\alpha^{0}$ & 10000011 & 131 & 116 \\
\hline 249 & $\alpha^{248}$ & $\alpha^{4}+\alpha^{3}+\alpha^{1}+\alpha^{0}$ & 00011011 & 27 & 214 \\
\hline
\end{tabular}




\begin{tabular}{|c|c|c|c|c|c|}
\hline 250 & $\alpha^{249}$ & $\alpha^{5}+\alpha^{4}+\alpha^{2}+\alpha^{1}$ & 00110110 & 54 & 244 \\
\hline 251 & $\alpha^{250}$ & $\alpha^{6}+\alpha^{5}+\alpha^{3}+\alpha^{2}$ & 01101100 & 108 & 234 \\
\hline 252 & $\alpha^{251}$ & $\alpha^{7}+\alpha^{6}+\alpha^{4}+\alpha^{3}$ & 11011000 & 216 & 168 \\
\hline 253 & $\alpha^{252}$ & $\alpha^{7}+\alpha^{5}+\alpha^{3}+\alpha^{2}+\alpha^{0}$ & 10101101 & 173 & 80 \\
\hline 254 & $\alpha^{253}$ & $\alpha^{6}+\alpha^{2}+\alpha^{1}+\alpha^{0}$ & 01000111 & 71 & 88 \\
\hline 255 & $\alpha^{254}$ & $\alpha^{7}+\alpha^{3}+\alpha^{2}+\alpha^{1}$ & 10001110 & 142 & 175 \\
\hline & $\alpha^{255}$ & $\alpha^{0}$ & 00000001 & 1 & \\
\hline
\end{tabular}

